\title{
Distributed Gaussian Polynomials as q-oscillator eigenfunctions
}

\author{
Hasan Karabulut \\ Rize University, \\ Faculty of Arts and Sciences, \\ Physics department, \\ 53100 Rize/TURKEY
}

\begin{abstract}
Karabulut and Sibert (J. Math. Phys. 38 (9), 4815 (1997)) have constructed an orthogonal set of functions from linear combinations of equally spaced Gaussians. In this paper we show that they are actually eigenfunctions of a q-oscillator in coordinate representation. We also reinterpret the coordinate representation example of q-oscillator given by Macfarlane as the functions orthogonal with respect to an unusual inner product definition. It is shown that the eigenfunctions in both q-oscillator examples are infinitely degenerate.
\end{abstract}

\section{INTRODUCTION}

Distributed Gaussians are a set of equally spaced Gaussians: $g_{n}(x)=\mathrm{e}^{-c^{2}(x-n)^{2}}$ where $(n=-\infty, \ldots \infty)$. A finite chain of them is often used in variational calculations as a basis set. They are a very flexible and efficient basis set often yielding very accurate variational results. Calculating the potential matrix elements are often the most difficult part of a variational calculation. Because of their compactness it is very easy to calculate potential energy matrix elements with a few point Gauss-Hermite quadrature very accurately which is a major advantage of using this basis.

We need orthogonal functions for variational calculations and the distributed Gaussians

are not orthogonal. In a 1997 paper Karabulut and Sibert[1] constructed a set of orthogonal functions from distributed Gaussians and they studied the underlying Gaussian quadrature. They called these functions distributed Gaussian polynomials (DG polynomials briefly). 
Their motivation was entirely practical and they were not looking for an algebraic structure behind these function. Later Karabulut[2] showed how to normalize them and used these functions to construct a Wannier function set from distributed Gaussians.

While searching for an operator that admits the DG polynomials as eigenfunctions the author came across a seminal paper by Macfarlane [3] in which he constructed a coordinate representation of his q-oscillator algebra. (the same q-oscillator was also studied by Biederharn [4] but he did not have the explicit coordinate representation example that Macfarlene gave). Eigenfunctions of Macfarlene were a linear combination of distributed Gaussians and it involved the q-binomial coefficients just like the DG polynomials. They looked similar to the DG polynomials but they were not the same. Following his example, the author constructed another coordinate representation of the q-oscillator algebra that yields the DG polynomials as eigenfunctions. The q-oscillator turns out to be a coordinate representation example of the Arik-Coon oscillator [5]. Macfarlane gave an inner product definition for his functions in term of Rogers-Szegö polynomials. I also found a simpler inner product definition for his functions and reinterpreted his results.

The outline of the paper is the following. In the second section I summarize basic results about the DG polynomials and discuss its links to Rogers-Szegö polynomials. In the third section I derive the DG polynomials from the q-oscillator algebra. In the fourth section I discuss Macfarlane's example to his q-oscillator and reinterpret its eigenfunctions. Finally, in the last section I give a summary and discussion.

\section{DISTRIBUTED GAUSSIAN POLYNOMIALS AND THEIR PROPERTIES}

We will mostly denote the Gaussians $\mathrm{e}^{-c^{2} x^{2}}$ as $q^{x^{2}}$ where $q=\mathrm{e}^{-c^{2}}$ and $c^{-1}$ is related to the width of the Gaussians. DG polynomials are defined as

$$
\Phi_{n}(x)=\sum_{k=0}^{n} C_{k}^{n}(-1)^{k} q^{-k / 2} q^{(x-k)^{2}} .
$$

The $C_{k}^{n}$ are the well known q-binomial coefficients

$$
C_{k}^{n}=\frac{(q, q)_{n}}{(q, q)_{k}(q, q)_{n-k}},
$$


where $(q, q)_{n}$ is defined as

$$
(q, q)_{n}=(1-q)\left(1-q^{2}\right) \ldots\left(1-q^{n}\right)
$$

and $(q, q)_{0}=1$. They satisfy the following orthogonality relation[2]:

$$
\int_{-\infty}^{\infty} \Phi_{n}(x) \Phi_{m}(x) \mathrm{d} x=\left\|\Phi_{n}(x)\right\|^{2} \delta_{n m},
$$

where the norm $\left\|\Phi_{n}(x)\right\|$ is given as

$$
\left\|\Phi_{n}(x)\right\|=\left(\frac{\pi}{2 c^{2}}\right)^{1 / 4} q^{-n / 2} \sqrt{(q, q)_{n}} .
$$

We will denote the normalized functions with lowercase $\phi$

$$
\phi_{n}=\frac{\Phi_{n}(x)}{\left\|\Phi_{n}(x)\right\|}=\frac{\alpha}{\sqrt{(q, q)}} \sum_{k=0}^{n} C_{k}^{n}(-1)^{k} q^{(n-k) / 2} q^{(x-k)^{2}},
$$

where

$$
\alpha=\left(\int_{-\infty}^{\infty} q^{2 x^{2}} \mathrm{~d} x\right)^{-1 / 2}=\left(\frac{2 c^{2}}{\pi}\right)^{1 / 4} .
$$

We defined $\alpha$ this way for later convenience.

Karabulut and Sibert(1] also found that the DG polynomials yield harmonic oscillator eigenfunctions in a particular limit as

$$
\lim _{c \rightarrow 0} \frac{\Phi_{n}(s / \sqrt{2} c)}{(-c / \sqrt{2})^{n}}=\mathrm{e}^{-s^{2} / 2} h_{n}(s),
$$

where $h_{n}(s)$ are the standard Hermite polynomials. We will refer to this limit later.

DG polynomials are related to the Stieltjes-Wigert polynomials. Let us write the orthogonality relation as follows

$$
\int_{-\infty}^{\infty} \Phi_{n}(x-s) \Phi_{m}(x-s) \mathrm{d} x=0,(n \neq m) .
$$

If we denote $u=q^{-2 x}$ then $\Phi_{n}(x-s)$ is written as

$$
\Phi_{n}(x-s)=\mathrm{e}^{-(\ln u)^{2} /(-4 \ln q)} u^{s} P_{n}(u ; s),
$$

where the polynomials $P_{n}(u ; s)$ are

$$
P_{n}(u ; s)=\sum_{k=0}^{n} C_{k}^{n}(-1)^{k} q^{(k+s)^{2}-k / 2} u^{k} .
$$


Then the orthogonality relation becomes

$$
\int_{-\infty}^{\infty} \mathrm{e}^{-(\ln u)^{2} /(-2 \ln q)} u^{2 s-1} P_{n}(u ; s) P_{m}(u ; s) \mathrm{d} x=0, \quad(n \neq m) .
$$

Evidently the polynomials $P_{n}(u ; s)$ are orthogonal with respect to the weight function

$$
W(u)=\mathrm{e}^{-(\ln u)^{2} /(-2 \ln q)} u^{2 s-1} .
$$

For $s=1 / 2$ the weight function is the lognormal distribution and the corresponding polynomials are known as the Stieltjes-Wigert polynomials. So the $P_{n}(u ; 1 / 2)$ are proportional to Stieltjes-Wigert polynomials.

The above connection to the Stieltjes Wigert polynomials were noted in Karabulut and Sibert[1]. Later Atakishiyev and Nagiyev[6] found a connection between the Stieltjes-Wigert polynomials and Rogers-Szegö polynomials through the Fourier transform which implied that DG polynomials are also connected the Rogers-Szegö polynomials. Here we note this connection.

We take the convention for the Fourier transform as

$$
f(\theta)=\int_{-\infty}^{\infty} \mathrm{e}^{\mathrm{i} 2 \pi \theta x} f(x) \mathrm{d} x
$$

Using the Parseval relation of the Fourier transforms

$$
\int_{-\infty}^{\infty} F^{*}(x) G(x) \mathrm{d} x=\int_{-\infty}^{\infty} F^{*}(\theta) G(\theta) \mathrm{d} \theta
$$

we write the eq. (4) as

$$
\int_{-\infty}^{\infty} \Phi_{n}^{*}(\theta) \Phi_{m}(\theta) \mathrm{d} \theta=\left(\frac{\pi}{2 c^{2}}\right)^{1 / 2} q^{-n}(q, q)_{n} \delta_{n m},
$$

where $\Phi_{n}(\theta)$ is the Fourier transform of $\Phi_{n}(x)$ given as

$$
\Phi_{n}(\theta)=\left(\frac{\pi}{c^{2}}\right)^{1 / 2} \mathrm{e}^{-(\pi / c)^{2} \theta^{2}} \sum_{k=0}^{n} C_{k}^{n}\left(-q^{-1 / 2} e^{i 2 \pi \theta}\right)^{k}
$$

The polynomials

$$
H_{n}(x)=\sum_{k=0}^{n} C_{k}^{n} x^{k}
$$


are known as Rogers-Szegö polynomials [6, 7, 8]. Using them the orthogonality relation is written as

$$
\int_{-\infty}^{\infty} H_{n}\left(-q^{-1 / 2} \mathrm{e}^{-\mathrm{i} 2 \pi \theta}\right) H_{n}\left(-q^{-1 / 2} \mathrm{e}^{\mathrm{i} 2 \pi \theta}\right) \mathrm{e}^{-2(\pi / c)^{2} \theta^{2}} \mathrm{~d} \theta=\left(\frac{c^{2}}{2 \pi}\right)^{1 / 2} q^{-n}(q, q)_{n} \delta_{n m} .
$$

A form of the Poisson summation formula reads

$$
\int_{-\infty}^{\infty} f(x) \mathrm{d} x=\int_{0}^{1}\left(\sum_{k=-\infty}^{\infty} f(x+k)\right) \mathrm{d} x
$$

which is valid when $\sum_{k=-\infty}^{\infty} f(x+k)$ exists (in our case it does). Using this eq. (19) is expressed as

$$
\int_{0}^{1} H_{n}\left(-q^{-1 / 2} \mathrm{e}^{-\mathrm{i} 2 \pi \theta}\right) H_{n}\left(-q^{-1 / 2} \mathrm{e}^{\mathrm{i} 2 \pi \theta}\right)\left(\sum_{k=-\infty}^{\infty} \mathrm{e}^{-2(\pi / c)^{2}(\theta+k)^{2}}\right) \mathrm{d} \theta=\left(\frac{c^{2}}{2 \pi}\right)^{1 / 2} q^{-n}(q, q)_{n} \delta_{n m} .
$$

The sum in parenthesis is periodic with period unity and it is a form of theta function. We can expand it in Fourier series. The Fourier coefficients can be calculated using the Poisson summation formula as

$$
\sum_{k=-\infty}^{\infty} \mathrm{e}^{-2(\pi / c)^{2}(\theta+k)^{2}}=\sqrt{\frac{c^{2}}{2 \pi}} \sum_{k=-\infty}^{\infty} q^{n^{2} / 2} \mathrm{e}^{\mathrm{i} 2 \pi n \theta}
$$

Using the Jacobi $\vartheta_{3}$ function[9] defined as

$$
\vartheta_{3}(\theta ; q)=\sum_{n=-\infty}^{\infty} q^{n^{2} / 2} \mathrm{e}^{\mathrm{i} n \theta}
$$

the orthogonality is written as follows:

$$
\int_{0}^{1} H_{n}\left(-q^{-1 / 2} \mathrm{e}^{-\mathrm{i} 2 \pi \theta}\right) H_{m}\left(-q^{-1 / 2} \mathrm{e}^{\mathrm{i} 2 \pi \theta}\right) \vartheta_{3}(2 \pi \theta ; q) \mathrm{d} \theta=q^{-n}(q, q)_{n} \delta_{n m} .
$$

This relation is the well known orthogonality of the Rogers-Szegö polynomials on the unit circle. Clearly it is the same thing as the orthogonality of the DG polynomials and one can be expressed in terms of the other. This is also of interest because Macfarlane [3] expressed orthogonality of his q-oscillator eigenfunctions in terms of orthogonality of Rogers Szegö polynomials on the unit circle. 


\section{DG POLYNOMIALS AS A Q-OSCILLATOR EIGENFUNCTIONS}

\section{A. Algebraic derivation of DG polynomials}

Lets define the translation operator $T^{s}$ as $T^{s}=\mathrm{e}^{s \frac{\partial}{\partial x}}$. It has the effect of shifting a function to the left by $s: T^{s} f(x)=f(x+s)$. We define the creation and destruction operators $\widehat{a}$ and $\widehat{a}^{\dagger}$ as

$$
\begin{aligned}
\widehat{a} & =\frac{1}{\sqrt{1-q}} T^{1 / 2}\left[q^{x+1 / 4}-T^{1 / 2}\right], \\
\widehat{a}^{\dagger} & =\frac{1}{\sqrt{1-q}}\left[q^{x+1 / 4}-T^{-1 / 2}\right] T^{-1 / 2}
\end{aligned}
$$

Our inner product is the usual one

$$
(f, g)=\int_{-\infty}^{\infty} f^{*}(x) g(x) \mathrm{d} x
$$

and the conjugate operator is defined as $(f, \widehat{O} g)=\left(\widehat{O}^{\dagger} f, g\right)$. According to this $\left(q^{x}\right)^{\dagger}=q^{x}$ and $\left(\frac{\partial}{\partial x}\right)^{\dagger}=-\frac{\partial}{\partial x}$ and $\widehat{a}^{\dagger}$ given above is the right one. The $\widehat{a}$ and $\widehat{a}^{\dagger}$ satisfy the commutation relation

$$
\widehat{a} \widehat{a}^{\dagger}-q \widehat{a}^{\dagger} \widehat{a}=1
$$

This is the commutation relation satisfied by the Arik-Coon oscillator[5].

We look for the eigenstates of the $\widehat{a}^{\dagger} \widehat{a}$

$$
\widehat{a}^{\dagger} \widehat{a} A_{n}(x)=\lambda_{n} A_{n}(x)
$$

We start from a 'ground state' $A_{0}(x)$ that satisfies $\widehat{a} A_{0}(x)=0$ which yields the functional equation

$$
A_{0}(x+1 / 2)=q^{(x+1 / 4)} A_{0}(x)
$$

If we put $A_{0}(x)=($ cons. $) w(x) q^{x^{2}}$ in this equation we get $w(x+1 / 2)=w(x)$. Therefore the normalized ground state is

$$
A_{0}(x)=\alpha_{w} w(x) q^{x^{2}},
$$


where $w(x)$ is any (in general complex) function satisfying $w(x+1 / 2)=w(x)$ periodicity condition and $\alpha_{w}$ is the normalization coefficient

$$
\alpha_{w}=\left(\int_{-\infty}^{\infty}|w(x)|^{2} q^{2 x^{2}} d x\right)^{-1 / 2} .
$$

For $w(x)=1$ we denote $\alpha_{w}$ as just $\alpha$ whose value is given in eq. (77). We choose normalization of our eigenfunctions as $\left(A_{n}, A_{n}\right)=1$ and taking $w(x)=1$ with this normalization will lead us to the normalized DG polynomials.

Next we build the states $\left(\widehat{a}^{\dagger}\right)^{n} A_{0}(x)$. Using the commutation relation one can easily show that if $\left(\widehat{a}^{\dagger}\right)^{n} A_{0}(x)$ is an eigenfunction of $\widehat{a}^{\dagger} \widehat{a}$ with the eigenvalue $\lambda_{n}$ then the $\left(\widehat{a}^{\dagger}\right)^{n+1} A_{0}(x)$ is an eigenfunction of the $\widehat{a}^{\dagger} \widehat{a}$ with eigenvalue $\lambda_{n+1}$ and one obtains a recursion relation for the eigenvalues

$$
\lambda_{n+1}=q \lambda_{n}+1 .
$$

Since $\widehat{a} A_{0}(x)=0$, then $A_{0}(x)$ is an eigenfunction of $\widehat{a}^{\dagger} \widehat{a}$ with the eigenvalue $\lambda_{0}=0$ and by induction it follows that all $\left(a^{\dagger}\right)^{n} A_{0}(x)$ are eigenfunctions. Using the recursion relation in eq. (33) and $\lambda_{0}=0$ we get the eigenvalues as

$$
\lambda_{n}=\frac{1-q^{n}}{1-q} .
$$

In exactly the same way that we do in solving the harmonic oscillator algebraically, we can easily obtain the following relations

$$
\begin{aligned}
\widehat{a} A_{n}(x) & =\sqrt{\lambda_{n}} A_{n-1}(x), \\
\widehat{a}^{\dagger} A_{n}(x) & =\sqrt{\lambda_{n+1}} A_{n+1}(x) .
\end{aligned}
$$

Then $A_{n}(x)$ can be written as

$$
A_{n}(x)=\sqrt{\frac{(1-q)^{n}}{(q, q)_{n}}}\left(\widehat{a}^{\dagger}\right)^{n} A_{0}(x) .
$$

Instead of applying $\widehat{a}^{\dagger} \mathrm{n}$ times, the following recursive relation is easier. Define $A_{n}(x)$ as

$$
A_{n}(x)=\frac{\alpha_{w} w(x)}{\sqrt{(q, q)_{n}}} \sum_{k=0}^{n} D_{k}^{n}(-1)^{k} q^{(n-k) / 2} q^{(x-k)^{2}} .
$$


Then if we apply $\widehat{a}^{\dagger} / \sqrt{\lambda_{n+1}}$ to obtain $A_{n+1}(x)$ and compare the coefficients we get the recursion relation for the $D_{k}^{n}$ coefficients. In this process $w(x)$ completely commutes with $\widehat{a}^{\dagger}$ because of periodicity: $T^{-1 / 2} w(x)=w(x-1 / 2)=w(x)$. The recursion relation we obtain is

$$
D_{k}^{n+1}=q^{k} D_{k}^{n}+D_{k-1}^{n}
$$

Together with the conditions $D_{0}^{0}=1, D_{-1}^{0}=0$ this completely determines the $D_{k}^{n}$. As can be shown easily, the q-binomial coefficients $C_{k}^{n}$ satisfy this recursion relation and the boundary conditions and therefore

$$
D_{k}^{n}=C_{k}^{n}=\frac{(q, q)_{n}}{(q, q)_{k}(q, q)_{n-k}}
$$

This shows that

$$
A_{n}(x)=\frac{\alpha_{w} w(x)}{\sqrt{(q, q)_{n}}} \sum_{k=0}^{n} C_{k}^{n}(-1)^{k} q^{(n-k) / 2} q^{(x-k)^{2}},
$$

are the normalized eigenfunctions and they reduce to the normalized DG polynomials $\phi_{n}(x)$ when $w(x)=1$.

Now let us see how the q-oscillator algebra reduces to harmonic oscillator algebra in the limit $q \rightarrow 0(c \rightarrow 0)$. The limit in equation (8) shows us how to do it. First define the new variable $z=c x$. With this variable, the $\widehat{a}$ will look like

$$
\widehat{a}=\frac{1}{\sqrt{1-\mathrm{e}^{-c^{2}}}} \mathrm{e}^{(c / 2) \partial / \partial z}\left[\mathrm{e}^{-c^{2} / 4} e^{-c z}-\mathrm{e}^{\frac{c}{2}(\partial / \partial z)}\right]
$$

and in the limit $c \rightarrow 0$ this reduces to

$$
\widehat{a} \rightarrow-\frac{1}{2} \frac{\partial}{\partial z}-z
$$

Similarly, $\widehat{a}^{\dagger}$ reduces to

$$
\widehat{a}^{\dagger} \rightarrow \frac{1}{2} \frac{\partial}{\partial z}-z
$$

which, together with $\widehat{a}$, are the destruction and creation operators for the harmonic oscillator problem

$$
\left(-\frac{1}{4} \frac{\partial}{\partial z^{2}}+z^{2}\right) \psi(z)=E \psi(z)
$$




\section{B. Further discussion on $w(x)$ degeneracy}

Although the algebraic solution seems flawless, it is still very surprising that the $A_{n}(x)$ are orthogonal for all the functions $w(x)$ satisfying $w(x+1 / 2)=w(x)$. Here we give a separate proof of it.

When we multiply two of our parent Gaussians $g_{n}(x)=q^{(x-n)^{2}}$ we get daughter Gaussians $G_{n}(x)=q^{2(x-n / 2)^{2}}$ as

$$
g_{n}(x) g_{m}(x)=q^{(n-m)^{2} / 2} G_{n+m}(x) .
$$

The parent Gaussians are centered at integers whereas the Daughter Gaussians are centered at both integers and half integers. Therefore the $\phi_{n}(x) \phi_{m}(x)$ product of the normalized DG polynomials

$$
\phi_{n}(x)=\frac{\alpha}{\sqrt{(q, q)_{n}}} \sum_{k=0}^{n} C_{k}^{n}(-1)^{k} q^{(n-k) / 2} q^{(x-k)^{2}},
$$

can be written as a linear combination of Daughter Gaussians as

$$
\phi_{n}^{*}(x) \phi_{m}(x)=\alpha^{2} \sum_{k=0}^{n+m} d_{k}^{n m} q^{2(x-k / 2)^{2}} .
$$

If we integrate this we should get $\delta_{n m}$ due to the orthogonality of normalized DG polynomials. The integrals $\int_{-\infty}^{\infty} q^{2(x-k / 2)^{2}} \mathrm{~d} x$ are independent of $k$ (just shift the integral by $k / 2$ ) and have the value $1 / \alpha^{2}$. Therefore we get the relation

$$
\sum_{k=0}^{n+m} d_{k}^{n m}=\delta_{n m}
$$

Now, the $A_{n}^{*}(x) A_{m}(x)$ product of the $A_{n}(x)$ functions

$$
A_{n}(x)=\frac{\alpha_{w} w(x)}{\sqrt{(q, q)_{n}}} \sum_{k=0}^{n} C_{k}^{n}(-1)^{k} q^{(n-k) / 2} q^{(x-k)^{2}},
$$

can be expressed as

$$
A_{n}^{*}(x) A_{m}(x)=\left|\alpha_{w}\right|^{2} \sum_{k=0}^{n+m} d_{k}^{n m}\left(|w(x)|^{2} q^{2(x-k / 2)^{2}}\right) .
$$

If we integrate this we get

$$
\int_{-\infty}^{\infty} A_{n}^{*}(x) A_{m}(x) \mathrm{d} x=\sum_{k=0}^{n+m} d_{k}^{n m}\left(\left|\alpha_{w}\right|^{2} \int_{-\infty}^{\infty}|w(x)|^{2} q^{2(x-k / 2)^{2}} \mathrm{~d} x\right) .
$$


The integral in parenthesis can be shifted by $k / 2$ as

$$
\int_{-\infty}^{\infty}|w(x)|^{2} q^{2(x-k / 2)^{2}} \mathrm{~d} x=\int_{-\infty}^{\infty}|w(x+k / 2)|^{2} q^{2 x^{2}} \mathrm{~d} x .
$$

Because of the periodicity of $w(x)$, we have $w(x+k / 2)=w(x)$ and all the integrals are independent of $k$ and they have the value $1 /\left|\alpha_{w}\right|^{2}$. This yields

$$
\int_{-\infty}^{\infty} A_{n}^{*}(x) A_{m}(x) \mathrm{d} x=\sum_{k=0}^{n+m} d_{k}^{n m}=\delta_{n m}
$$

which follows from eq.(49). Therefore the orthogonality holds for any $w(x)$ satisfying the periodicity requirement.

We actually found more than the DG polynomials from the algebraic treatment. We found and infinite set of orthogonal functions.

\section{A DIFFERENT INTERPRETATION OF Q-OSCILLATOR EXAMPLE OF MACFARLANE}

In a seminal and widely cited paper Macfarlane constructed a different coordinate representation of the q-oscillator. His definition of the creation and destruction operators are

$$
\begin{gathered}
\widehat{b}=\mathrm{e}^{2 x}-\mathrm{e}^{x} \mathrm{e}^{s(\partial / \partial x)}, \\
\widehat{b}^{\dagger}=\mathrm{e}^{-2 x}-\mathrm{e}^{s(\partial / \partial x)} \mathrm{e}^{-x} .
\end{gathered}
$$

Obviously, according to the usual definition of the inner product in eq.(27) the $\hat{b}^{\dagger}$ is not right. Macfarlane himself goes on to construct eigenstates of $\widehat{b}^{\dagger} \widehat{b}$ without discussing the inner product and orthogonality relation first. After finding the eigenfunctions he states inner product and orthogonality relations in terms of orthogonality of Rogers-Szegö polynomials on the unit circle. The functions we find do not agree with the functions he found and apparently his formula for the eigenfunctions contains some error. Therefore we redo the problem with a different inner product definition here.

We define the inner product as 


$$
\begin{aligned}
(f, g) & =\int_{-\infty}^{\infty}\left(\widehat{P} f^{*}(x)\right) g(x) \mathrm{d} x \\
& =\int_{-\infty}^{\infty} f^{*}(-x) g(x) \mathrm{d} x
\end{aligned}
$$

where $\widehat{P}$ is the usual parity operator. For an operator $\widehat{O}$, its conjugate $\widehat{O}^{\dagger}$ is defined by the relation

$$
(f, \widehat{O} g)=\left(\widehat{O}^{\dagger} f, g\right)
$$

The $\widehat{b}^{\dagger}$ expression in eq.(56) is right if one defines the inner product this way because $\left(\mathrm{e}^{x}\right)^{\dagger}=\mathrm{e}^{-x}$ and $\left(\frac{d}{d x}\right)^{\dagger}=\frac{d}{d x}$. Notice that this definition of the inner product does not satisfy $(f, f) \geq 0$ condition. But we will not use this property of the inner product in our development. We will use the conjugacy definition in eq.(59) in a few places.

In order to obtain functions as a linear combination of the Gaussians centered at nonnegative integers we change the variable $x=-c^{2}(y+1 / 4)$ and $s=-c^{2} / 2$ and we take $q=\mathrm{e}^{-c^{2}}$ as before. We will also divide $\widehat{b}$ and $\widehat{b}^{\dagger}$ by $\sqrt{q(1-q)}$ which does not change the eigenfunctions (only eigenvalues), but in the limit of the harmonic oscillator $(q \rightarrow 1)$ it helps to get the right results. The new $\widehat{b}$ and $\widehat{b}^{\dagger}$ are

$$
\begin{aligned}
\widehat{b} & =\left(q^{2 y+1 / 2}-q^{y+1 / 4} T^{1 / 2}\right) / \sqrt{q(1-q)}, \\
\hat{b}^{\dagger} & =\left(q^{-2 y+1 / 2}-T^{1 / 2} q^{-y+1 / 4}\right) / \sqrt{q(1-q)},
\end{aligned}
$$

where $T^{1 / 2}=\mathrm{e}^{(\partial / \partial y) / 2}$. They satisfy the commutation relation

$$
\widehat{b}^{\dagger} \widehat{b}-q \widehat{b}^{\dagger}=1
$$

Note that this is somewhat different than the commutation relation in eq.(28).

Again we want to find the eigenfunctions of the operator $\hat{b}^{\dagger} \hat{b}$

$$
\widehat{b}^{\dagger} \widehat{b} B_{n}(y)=\lambda_{n} B_{n}(y)
$$

We again start from a ground state that satisfies $\widehat{b} B_{0}(y)=0$ (hence $\left.\lambda_{0}=0\right)$. $\widehat{b} B_{0}(y)=0$ yields the same functional equation that $A_{0}(x)$ satisfy

$$
B_{0}(y+1 / 2)=q^{y+1 / 4} B_{0}(y)
$$


which we already know has the solution $B_{0}(y)=\alpha_{w} w(y) q^{y^{2}}$ where $w(y)$ satisfies the $w(y+$ $1 / 2)=w(y)$ periodicity condition. The infinite degeneracy of the states appear here too.

Next we build the unnormalized states $\left(\widehat{b}^{\dagger}\right)^{n} B_{0}(y)$. Using the commutation relation we can easily show that if the $\left(\widehat{b}^{\dagger}\right)^{n} B_{0}$ is an eigenfunction of $\widehat{b}^{\dagger} \widehat{b}$ with an eigenvalue $\lambda_{n}$ then $\left(\widehat{b}^{\dagger}\right)^{n+1} B_{0}$ is an eigenfunction with the eigenvalue $\lambda_{n+1}$ where $\lambda_{n+1}$ is related to the $\lambda_{n}$ as

$$
q \lambda_{n+1}=\lambda_{n}-1
$$

Since $B_{0}(y)$ is an eigenfunction, by induction all $\left(\widehat{b}^{\dagger}\right)^{n} B_{0}$ are eigenfunctions too. Starting from $\lambda_{0}=0$, this recursion relation yields

$$
\lambda_{n}=-q^{-n}\left(\frac{1-q^{n}}{1-q}\right) .
$$

Notice that the eigenvalues are negative.

To obtain relations similar to eqs.(35],36) We form the inner product

$$
\lambda_{n}\left(B_{n}, B_{n}\right)=\left(B_{n}, \widehat{b}^{\dagger} \widehat{b} B_{n}\right)=\left(\widehat{b} B_{n}, \widehat{b} B_{n}\right)
$$

Taking $\widehat{b} B_{n}=\mu_{n} B_{n-1}$ we get

$$
\lambda_{n}=\left|\mu_{n}\right|^{2} \frac{\left(B_{n-1}, B_{n-1}\right)}{\left(B_{n}, B_{n}\right)} .
$$

Since $\left|\mu_{n}\right|^{2}$ is positive and $\lambda_{n}$ is negative, the $\left(B_{n-1}, B_{n-1}\right)$ and $\left(B_{n}, B_{n}\right)$ must have opposite signs. This looks surprising but we should remember that in our definition of the inner product the $(f, f)$ can be negative. Therefore we will take our normalization as

$$
\left(B_{n}, B_{n}\right)=(-1)^{n}
$$

With this normalization we obtain $\mu_{n}=\sqrt{-\lambda_{n}}$ :

$$
\widehat{b} B_{n}=\sqrt{-\lambda_{n}} B_{n-1}
$$

Starting from

$$
\lambda_{n+1} B_{n+1}=\hat{b}^{\dagger} \widehat{b} B_{n+1}=\sqrt{-\lambda_{n+1}} \hat{b}^{\dagger} B_{n},
$$


we also obtain

$$
\widehat{b}^{\dagger} B_{n}=-\sqrt{-\lambda_{n+1}} B_{n+1}
$$

This result is also a little unusual because of the sign in front. It is a consequence of negative $\lambda_{n}$ eigenvalues.

Since we started from an unusual inner product definition there might be doubts on orthogonality of the $B_{n}$. Consider the inner product

$$
\left(B_{m}, \widehat{b}^{\dagger} \widehat{b} B_{n}\right)=\left(\widehat{b}^{\dagger} \widehat{b} B_{m}, B_{n}\right)
$$

Using $\widehat{b}^{\dagger} \widehat{b} B_{k}=\lambda_{k} B_{k}$ we get

$$
\left(\lambda_{n}-\lambda_{m}\right)\left(B_{m}, B_{n}\right)=0
$$

which yields $\left(B_{m}, B_{n}\right)=0$ when $m \neq n$. We added this common proof to emphasize that the orthogonality does not depend on the $(f, f) \geq 0$ property of the usual inner products. We just used the conjugacy relation eq.(59) in this proof of orthogonality. But unlike the case of usual definition inner product given in eq.(27),$(f, f)=0$ does not imply $f=0$ when we don't have $(f, f) \geq 0$ property.

To complete the discussion we obtain the eigenfunctions $B_{n}(y)$. It makes things easier to figure out the coefficient of Gaussian centered at $\operatorname{zero}\left(q^{y^{2}}\right)$ first. The $\hat{b}^{\dagger}$

$$
\widehat{b}^{\dagger}=\left(q^{-2 y+1 / 2}-T^{1 / 2} q^{-y+1 / 4}\right) / \sqrt{q(1-q)}
$$

has two parts that do different things. When $q^{-2 y+1 / 2}$ acts on $q^{(y-k)^{2}}$ it produces the next Gaussian $q^{(y-k-1)^{2}}$ and multiplies it with a constant. When $T^{1 / 2} q^{-y+1 / 4}$ act on $q^{(y-k)^{2}}$ it gives $q^{(y-k)^{2}}$ back and multiplies with a constant. Here are the precise relations

$$
\begin{aligned}
q^{-2 y+1 / 2} q^{(y-k)^{2}} & =q^{-2 k-1 / 2} q^{(y-k-1)^{2}}, \\
T^{1 / 2} q^{-y+1 / 4} q^{(y-k)^{2}} & =q^{-k} q^{(y-k)^{2}} .
\end{aligned}
$$

From the second relation we have $T^{1 / 2} q^{-y+1 / 4} q^{y^{2}}=q^{y^{2}}$ which means this operator leaves $q^{y^{2}}$ as it is. The other operator $q^{-2 y+1 / 2}$ creates $k=1$ Gaussian $\left(q^{(y-1)^{2}}\right)$ from it. Since we produce higher eigenfunctions by applying $-\widehat{b}^{\dagger} / \sqrt{-\lambda_{n}}$ successively, after each application 
the coefficient of $k=0$ Gaussian changes by a factor $1 / \sqrt{q(1-q)\left(-\lambda_{n}\right)}$. We denote the coefficient of $k=0$ Gaussian in $B_{n}(y)$ by $\zeta_{n}$ and it should be

$$
\zeta_{n}=\alpha_{w} \frac{1}{\sqrt{q^{n}(1-q)^{n}}} \frac{1}{\sqrt{\left(-\lambda_{1}\right)\left(-\lambda_{2}\right) \ldots\left(-\lambda_{n}\right)}}=\alpha_{w} \frac{q^{n(n-1) / 4}}{\sqrt{(q, q)_{n}}} .
$$

Now let us take the $B_{n}(y)$ of the form

$$
B_{n}(y)=w(x) \zeta_{n} \sum_{k=0}^{n} E_{k}^{n} q^{(y-k)^{2}}
$$

where $E_{0}^{n}=1$ by construction. We can use Eq. (172) to generate a recursion relation for $E_{k}^{n}$ as before. To show a different and easier way of doing things we will use eq.(70) relation this time. The $\widehat{b}$ has the effect of shifting each Gaussian to the left by one unit

$$
\widehat{b} q^{(y-k)^{2}}=-\frac{q^{k-1 / 2}\left(1-q^{k}\right)}{\sqrt{q(1-q)}} q^{(y-k+1)^{2}},
$$

and it also destroys the leftmost Gaussian (the $k=0$ Gaussian centered at zero). Therefore the equality

$$
\widehat{b}\left[\left(\zeta_{n} E_{k}^{n}\right) q^{(y-k)^{2}}\right]=\sqrt{-\lambda_{n}}\left[\left(\zeta_{n-1} E_{k-1}^{n-1}\right) q^{(y-k+1)^{2}}\right]
$$

should hold for each Gaussian. This yields the recursion relation for the $E_{k}^{n}$ as

$$
E_{k}^{n}=-E_{k-1}^{n-1}\left(\frac{1-q^{n}}{1-q^{k}}\right) q^{-n-k+3 / 2}
$$

Together with the condition $E_{0}^{n}=1$ this is enough information to solve the $E_{k}^{n}$. The quotient in parenthesis tells us that the q binomial coefficients $C_{k}^{n}$ are involved. If we set $E_{k}^{n}=(-1)^{k} C_{k}^{n} q^{u(n, k)}$ we get the recursion relation for $u(n, k)$ as

$$
u(n, k)-u(n-1, k-1)=-n-k+3 / 2
$$

Together with the condition $u(n, 0)=0$ (which follows from $E_{0}^{n}=1$ ) this is uniquely solved as $u(n, k)=-n k+k / 2$. Therefore the $B_{n}(y)$ should be

$$
B_{n}(y)=\alpha_{w} w(x) \frac{q^{n(n-1) / 4}}{\sqrt{(q, q)_{n}}} \sum_{k=0}^{n} C_{k}^{n}(-1)^{k} q^{-(n-1 / 2) k} q^{(y-k)^{2}}
$$


This formula does not agree with the result of Macfarlane even after setting $c^{2}=-2 s$ and $y=-1 / 4+x / 2 s$ back in $B_{n}(y)$ above. To be sure that we have the right formula we have checked numerically if the $B_{n}(y)$ satisfy the orthogonality relation

$$
\int_{-\infty}^{\infty} B_{n}(-y) B_{m}(y) \mathrm{d} y=(-1)^{n} \delta_{n m}
$$

They satisfy it perfectly and we are sure that we have the right formula. Apparently Macfarlane's paper contains an error.

The harmonic oscillator limit is as straightforward as it is in DG polynomials case. We change variable $y=z / c$ and take the limit $c \rightarrow 0$ which yields

$$
\begin{gathered}
\widehat{b} \rightarrow z-\frac{1}{2} \frac{\partial}{\partial z} \\
\widehat{b}^{\dagger} \rightarrow-z-\frac{1}{2} \frac{\partial}{\partial z} .
\end{gathered}
$$

Then $\widehat{b}^{\dagger} \widehat{b} B_{n}=\lambda_{n} B_{n}$ reduces to $\left(\lambda_{n} \rightarrow-n\right.$ in this limit $)$

$$
\left(-\frac{1}{4} \frac{\partial^{2}}{\partial z^{2}}+z^{2}\right) \Psi_{n}=(n+1 / 2) \Psi_{n}
$$

The fact that the inner product is defined differently makes no difference in this limit because the harmonic oscillator eigenfunctions are either even or odd. The harmonic oscillator eigenfunctions satisfy the normalization condition

$$
\int \Psi_{n}(-y) \Psi_{m}(y) \mathrm{d} y=(-1)^{n} \delta_{n m}
$$

as can be verified easily using parity of the wave functions.

Now, just as the DG polynomials, the orthogonality of the $B_{n}(y)$ can be expressed as an orthogonality relation of the Rogers-Szegö polynomials on the unit circle. We will take $w(x)=1$ and $\alpha_{w}=\alpha$ for this. By Fourier transforming the orthogonality relation eq. (85) we get the relation

$$
\int_{-\infty}^{\infty} B_{n}^{*}(-\theta) B_{m}(\theta) \mathrm{d} \theta=(-1)^{n} \delta_{n m}
$$

where $B_{n}(\theta)$ is the Fourier transform of the $B_{n}(y)$ :

$$
B_{n}(\theta)=\left(\frac{\pi}{c^{2}}\right)^{1 / 2} \zeta_{n} \mathrm{e}^{-(\pi / c)^{2} \theta^{2}} \sum_{k=0}^{n} C_{k}^{n}\left(-q^{-(n-1 / 2)} \mathrm{e}^{\mathrm{i} 2 \pi \theta}\right)^{k} .
$$


Here $\theta$ is the Fourier transform variable just as before. Using the Poisson summation formula in eq. (20) the orthogonality relation can be expressed as

$$
\int_{0}^{1} H_{n}\left(-q^{-(n-1 / 2)} \mathrm{e}^{\mathrm{i} 2 \pi \theta}\right) H_{m}\left(-q^{-(m-1 / 2)} \mathrm{e}^{\mathrm{i} 2 \pi \theta}\right)\left(\sum_{k=-\infty}^{\infty} \mathrm{e}^{-2(\pi / c)^{2}(\theta+k)^{2}}\right) \mathrm{d} \theta=\frac{c^{2}}{\pi} \frac{(-1)^{n}}{\zeta_{n}^{2}} \delta_{n m} .
$$

Setting the sum in parenthesis from the eq. (22) and (23) we obtain

$$
\int_{0}^{1} H_{n}\left(-q^{-(n-1 / 2)} \mathrm{e}^{\mathrm{i} 2 \pi \theta}\right) H_{m}\left(-q^{-(m-1 / 2)} \mathrm{e}^{\mathrm{i} 2 \pi \theta}\right) \vartheta_{3}(2 \pi \theta ; q) \mathrm{d} \theta=q^{-n(n-1) / 2}(q, q)_{n}(-1)^{n} \delta_{n m} .
$$

This is again some form of the orthogonality relation of the Rogers-Szegō polynomials on the circle. This is a new set of orthogonality relations and we are not aware of its existence in mathematical literature.

\section{SUMMARY}

In this study we found that the DG polynomials that Karabulut and Sibert discovered before are actually eigenfunctions of coordinate representation of the Arik-Coon q-oscillator. We derived the DG polynomials from q-oscillator algebra. We also indicated that orthogonality of the DG polynomials can be cast into orthogonality of the Rogers-Szegö polynomials on the unit circle and the two are equivalent.

We showed that the example given by Macfarlane can be interpreted with an unusual inner product definition and we constructed the corresponding orthogonal functions. Their orthogonality can be recast into a form of orthogonality relation for the Rogers Szegö polynomials on the unit circle. We were not able to find this result in mathematical literature and it is probably a new result.

A very interesting result of this work is that the eigenstates of the q-oscillators we solved turned out to be infinitely degenerate. We know that algebraic solution of the one dimensional harmonic oscillator is nondegenerate. In the usual algebraic solution of the harmonic oscillator we base our arguments on the commutation relations and the algebra has nothing in it that implies nondegenerate states. But the ground state $\widehat{a} \phi_{0}=0$ yields a unique solution for the harmonic oscillator because it is a differential equation and nondegeneracy of the 
excited states follows from this. For our q-oscillator examples we have a first order difference equation for the $\widehat{a} \phi_{0}=0$ and such equations together with the boundary conditions do not uniquely define a function. It defines a function on all real axis if its values in a $1 / 2$ wide interval are known.

Finally, the freedom to choose $w(x)$ arbitrarily gives us possibility of constructing orthogonal function sets more general than the DG polynomials. Consider a set of functions $w_{0}(x), w_{1}(x), w_{2}(x) \ldots$ satisfying the periodicity condition $w_{n}(x+1 / 2)=w_{n}(x)$ $(n=0,1,2, \ldots)$ and the orthogonality relation

$$
\int_{-\infty}^{\infty} w_{n}^{*}(x) w_{m}(x) q^{2 x^{2}} \mathrm{~d} x=\delta_{n m} .
$$

Then the set of functions

$$
\Gamma_{n m}(x)=\frac{\alpha\left(w_{n}\right)}{\alpha} w_{n}(x) \phi_{m}(x)
$$

will satisfy an orthogonality relation of the form

$$
\int_{-\infty}^{\infty} \Gamma_{n m}^{*}(x) \Gamma_{i j}(x) \mathrm{d} x=\delta_{n i} \delta_{m j} .
$$

Here $\alpha\left(w_{n}\right)$ is the $\alpha_{w}$ for $w=w_{n}(x)$ given in eq.(32) and $\alpha$ is given in eq.(7). This gives us much freedom to construct orthogonal function sets useful as basis sets in variational calculations. This possibility should be investigated in future research.

\section{Acknowledgments}

In a private communication Prof. R. G. Littlejohn suggested to the author that there must be an operator that admits the DG polynomials as eigenfunctions, or equivalently, there must be an algebraic structure behind them. Although his insightful comments and suggestions are entirely responsible for the creation of the work presented here, he kindly declined to coauthor this paper on the grounds that his contribution was minimal. The author is indebted to Professor Robert G. Littlejohn for suggesting the problem addressed in this paper.

[1] H. Karabulut and E. L. Sibert III (1997), J. Math. Phys. 38 (9), 4815 
[2] H. Karabulut (2005), J. Math. Phys., 46, 073504

[3] A. J. Macfarlane (1989), J. Phys. A:Math. Gen. 22, 4581

[4] L. C. Biedenharn (1989) J. Phys. A:Math. Gen., 22, L873

[5] M. Arik, D.D. Coon (1976), J. Math. Phys. 17 (4), 524

[6] N. M. Atakishiyev (1994), Sh. M. Nagiyev, J. Phys. A:Math. Gen., 27, L611

[7] G. Szegō (1991), Orthogonal polynomials Vol 23 (Providence, RI: AMS Colloquium Publications)

[8] G. E. Andrews (1976), The theory of partitions, Encyclopedia of Mathematics and its applications (Reading, MA: Addison-Wesley)

[9] E. T. Whittaker, G. N. Watson (1969), A Course of Modern Analysis(Cambridge, MA:Cambridge University Press) 